\title{
One-year outcome in patients with idiopathic normal-pressure hydrocephalus: comparison of lumboperitoneal shunt to ventriculoperitoneal shunt
}

\author{
Masakazu Miyajima, MD, PhD, ${ }^{1}$ Hiroaki Kazui, MD, PhD, ${ }^{2}$ Etsuro Mori, MD, $\mathrm{PhD},{ }^{3}$ and \\ Masatsune Ishikawa, MD, PhD, ${ }^{4}$ on behalf of the SINPHONI-2 Investigators \\ 1Department of Neurosurgery, Juntendo University Graduate School of Medicine, Tokyo; '2Department of Psychiatry, Osaka \\ University Graduate School of Medicine, Suita-city, Osaka; ${ }^{3}$ Department of Behavioral Neurology and Cognitive Neuroscience, \\ Tohoku University Graduate School of Medicine, Sendai-city, Miyagi; and ${ }^{4}$ Normal-Pressure Hydrocephalus Centre, Otowa \\ Hospital, Kyoto-city, Kyoto, Japan
}

OBJECTIVE Idiopathic normal pressure hydrocephalus (iNPH) is treated with cerebrospinal fluid shunting, and implantation of a ventriculoperitoneal shunt (VPS) is the current standard treatment. The objective of this study was to compare the efficacy and safety of VPSs and lumboperitoneal shunts (LPSs) for patients with iNPH.

METHODS The authors conducted a prospective multicenter study of LPS use for patients with iNPH. Eighty-three patients with iNPH (age 60 to 85 years) who presented with ventriculomegaly and high-convexity and medial subarachnoid space tightness on MR images were recruited from 20 neurological or neurosurgical centers in Japan between March 1 , 2010, and October 19, 2011. The primary outcome was the modified Rankin Scale (mRS) score 1 year after surgery, and the secondary outcome included scores on the iNPH grading scale (iNPHGS). A previously conducted VPS cohort study with the same inclusion criteria and primary and secondary end points was used as a historical control.

RESULTS The proportion of patients who achieved a favorable outcome (i.e., improvement of at least 1 point in their mRS score) was $63 \%(95 \% \mathrm{Cl} 51 \%-73 \%)$ and was comparable to values reported with VPS implantation $(69 \%, 95 \% \mathrm{Cl}$ $59 \%-78 \%)$. Using the iNPHGS, the 1-year improvement rate was $75 \%(95 \% \mathrm{Cl} 64 \%-84 \%)$ and was comparable to the rate found in the VPS study $(77 \%, 95 \% \mathrm{Cl} 68 \%-84 \%)$. The proportion of patients experiencing serious adverse events (SAEs) and non-SAEs did not differ significantly between the groups at 1 year after surgery (SAEs: 19 [22\%] of 87 LPS patients vs 15 [15\%] of 100 VPS patients, $p=0.226$; non-SAEs: 24 [27.6\%] LPS patients vs 20 [20\%] VPS patients, $p=$ $0.223)$. However, shunt revisions were more common in LPS-treated patients than in VPS-treated patients (6 [7\%] vs 1 $[1 \%])$.

CONCLUSIONS The efficacy and safety rates for LPSs with programmable valves are comparable to those for VPSs for the treatment of patients with iNPH. Despite the relatively high shunt failure rate, an LPS can be the treatment of choice because of its minimal invasiveness and avoidance of brain injury.

http://thejns.org/doi/abs/10.3171/2015.10.JNS151894

KEY WORDS cerebrospinal fluid; functional status; magnetic resonance imaging; subarachnoid space; ventriculomegaly; hydrocephalus

I DIOPATHIC normal pressure hydrocephalus (iNPH), which is a disease of uncertain etiology that characteristically afflicts the elderly, is treated with cerebrospinal fluid (CSF) shunting. iNPH is characterized by cognitive decline, gait and balance impairments, and urinary incontinence. ${ }^{2}$ Recent reports showing prevalence rates of $0.51 \%-2.9 \%$ in community-dwelling elderly people have suggested that $\mathrm{iNPH}$ is more common than previously thought. ${ }^{3,10-12,29}$

Ventriculoperitoneal shunt (VPS) implantation is the current standard treatment for patients with iNPH. ${ }^{5}$ A previous multicenter, prospective cohort study (i.e., Study of Idiopathic Normal Pressure Hydrocephalus on Neurological Improvement [SINPHONI]), in which 100 patients

ABBREVIATIONS ADL = activity of daily living; $\mathrm{BMI}=$ body mass index; $\mathrm{CSF}=$ cerebrospinal fluid; $\mathrm{DESH}=$ disproportionately enlarged subarachnoid space hydrocephalus; FAS = full analysis set; iNPH = idiopathic normal pressure hydrocephalus; iNPHGS = iNPH grading scale; LOCF = last observation carried forward; LPS $=$ lumboperitoneal shunt; MMSE = Mini-Mental State Examination; $m R S$ = modified Rankin Scale; PPS = per protocol set; $S A E$ = serious adverse event; $S A S=$ safety analysis set; SINPHONI = Study of Idiopathic Normal Pressure Hydrocephalus on Neurological Improvement; TUG = Timed Up and Go; VPS = ventriculoperitoneal shunt. SUBMITTED August 12, 2015. ACCEPTED October 15, 2015.

INCLUDE WHEN CITING Published online February 12, 2016; DOI: 10.3171/2015.10.JNS151894. 
were enrolled between November 2004 and November 2006, showed that specific MRI features in patients with iNPH, i.e., tight high-convexity and medial subarachnoid spaces and ventriculomegaly, which is defined as disproportionately enlarged subarachnoid space hydrocephalus (DESH), were associated with high responsiveness to VP shunting. ${ }^{8}$ However, to date, there have been no randomized controlled trials or systematic or prospective studies of lumboperitoneal shunt (LPS) implantation for the treatment of iNPH. Thus, the international iNPH guidelines suggest that LPSs can only be considered for patients with a preexisting seizure or other relative contraindication to VPS implantation. ${ }^{5}$ In addition, LPSs have not generally been favored by neurosurgeons because of high failure rates and the possibility of symptomatic overdrainage. ${ }^{6,16,31,33}$ However, the risk of symptomatic intraparenchymal hematoma from ventricular catheter placement, which is approximately $1 \%$ in patients with iNPH, is never present with LPS use, as LPSs avoid damage to the brain induced by punctures. ${ }^{7}$ There has been an increase in the use of LPSs rather than VPSs for the treatment of iNPH, particularly in Japan. ${ }^{15,26}$ However, in western countries, VPSs are used to treat iNPH in most cases because currently there is no established evidence to support using LPSs for this purpose. The long-term outcome of patients with iNPH who receive an LPS with a programmable valve remains to be determined. Therefore, we conducted a prospective multicenter trial to assess LPS implantation in patients with iNPH (SINPHONI-2), which integrated a 3-month randomized controlled trial (i.e., RCT phase) comparing LPS implantation to conservative therapy and a 12-month extension study (i.e., extension phase) in which all subjects received an LPS and were examined over the 12 months following implantation. The short-term and long-term beneficial effects of LPS use were obvious and have been reported previously..$^{18}$ In this paper, we present in further detail the long-term outcome of patients who received an LPS during the SINPHONI-2 trial and a comparative analysis of the results from the SINPHONI and SINPHONI-2 trials, which used common inclusion criteria and primary and secondary end points. The goal of this pooled analysis was to compare the efficacy and safety of VPS and LPS implantation in patients with iNPH specified as DESH.

\section{Methods \\ Study Design}

The SINPHONI-2 (registered with the University Hospital Medical Information Network Clinical Trials registry: UMIN000002730) protocol has been described in detail elsewhere. ${ }^{18}$ In brief, it was a prospective multicenter trial of LPS placement in patients with iNPH that integrated a 3-month randomized controlled phase, in which parallel groups received an LPS or conservative therapy, and a long-term phase, in which all participants received an LPS and were assessed for a 12-month period after surgery. The primary measure of efficacy for the 3-month randomized controlled phase was favorable outcome, which was defined as an improvement of 1 point or more on the modified Rankin Scale $(\mathrm{mRS})^{32}$ at 3 months after randomization, and the long-term end point was the same favorable outcome at 12 months after surgery. In contrast, the SINPHONI study was a prospective cohort study that was designed to test the safety and efficacy of VPS implantation in patients with iNPH. The study protocol was identical to that of SINPHONI-2 except that patients with severe vertebral degenerative diseases or spinal canal stenosis were excluded, and shunt surgery was postponed for 3 months in patients who were allocated to the control arm. SINPHONI-2 was conducted in compliance with the Guidelines for Good Clinical Practice and the Declaration of Helsinki (2002) of the World Medical Association. The study protocol was approved by the institutional review board at each site, and all patients (or their representative when applicable) provided written informed consent for participation. Twenty centers in Japan were involved in this study (Appendix). An independent committee monitored all clinical and imaging data, data related to safety issues, and protocol compliance via a web-based case report system.

\section{Patients}

Candidates for the SINPHONI-2 study were patients with suspected iNPH. The following inclusion criteria were adopted: 1) patient age between 60 and 85 years; 2) triad of symptoms, which were measurable on the iNPH grading scale (iNPHGS); ${ }^{21} 3$ ) both ventriculomegaly with an Evans' index $>0.3$ and high-convexity and medial subarachnoid space tightness on coronal MR images; ${ }^{19} 4$ ) absence of disorders known to produce ventriculomegaly; and 5) normal CSF content and pressure and a negative Queckenstedt's test. ${ }^{30}$ All patients who exhibited severe vertebral degenerative diseases or spinal canal stenosis on plain radiograph or MR images were excluded.

\section{Procedure}

Eligible patients were screened and selected by either neurologists or neurosurgeons and preregistered on the web system. Patients who met all the above criteria were enrolled. Then, participants were randomly assigned to either the immediate-shunt group (i.e., immediate group) or the postponed-shunt group (i.e., postponed group). Patients in the immediate group underwent LPS implantation within 1 month after randomization. They were evaluated before and 3, 6, and 12 months after surgery. Patients in the postponed group were instructed only to perform physical exercises (i.e., conservative therapy) for 3 months after randomization and were evaluated before and after the conservative therapy. After this therapy, they also underwent LPS implantation and were evaluated 3, 6, and 12 months after surgery.

LPS implantation was conducted under general anesthesia using a Codman-Hakim programmable valve with a Siphon-Guard (Codman and Shurtleff, Johnson and Johnson Inc.). Initial pressure for the shunt system was set before surgery according to the height and weight of the patient. ${ }^{25}$ Valve pressure was readjusted in 1- to $3-\mathrm{cm} \mathrm{H}_{2} \mathrm{O}$ intervals afterward, as required. Shunt function was assessed at each institution whenever needed. In addition, shunt function was checked when there was no improvement in clinical symptoms or when tight high-convexity 
and medial subarachnoid spaces, enlarged sylvian fissures, or acute callosal angles were observed.

\section{Outcome Measures}

The primary outcome measure was mRS. Secondary outcome measures included iNPHGS score, ${ }^{21}$ the 3-minute Timed Up and Go (TUG) test, ${ }^{27}$ and the Mini-Mental State Examination (MMSE). These evaluations and tests were performed by specialists other than the attending neurosurgeons, including neurologists, psychiatrists, clinical psychologists, and physical therapists. The primary end point was an improvement of 1 or more points in the patient's mRS score at 1 year after surgery (i.e., favorable outcome). A positive response to LPS implantation (i.e., shunt response) was defined as an improvement of more than 1 point in the $\mathrm{mRS}$ score at any evaluation point within 1 year. According to the protocol, if there was no significant difference in the improvement in activities of daily living (ADL) between the immediate and postponed groups at 12 months after shunt surgery, the 2 groups were combined. The utility of the LPS was compared with that of the VPS using the SINPHONI study data as a historical control.

Safety assessments included the recording of all adverse events throughout the study, regardless of whether they were related to treatment. A serious adverse event (SAE) was defined as 1 of the following: 1) death; 2) an event that could result in death; 3 ) an event for which treatment required hospitalization, admittance to a clinic, or an extension of the hospitalization period; and 4) an event that could result in morbidity.

\section{Statistical Analysis}

The full analysis set (FAS) was defined as all patients who underwent intervention and who had at least 1 valid postbaseline assessment of mRS score, whereas the per protocol set (PPS) was defined as all patients in the FAS excluding those with major protocol deviations. We used the PPS for all efficacy analyses, which made the data set compatible with that of the SINPHONI study. When mRS or iNPHGS scores were missing at any follow-up, scores were inputted using the "last observation carried forward" (LOCF) if the underlying assumption was justified. Adverse events were analyzed based on the safety analysis set (SAS), in which all patients who received an LPS were included. If there was no significant difference in the primary outcome measure between the groups at 12 months after surgery, the 2 groups were combined as a single LPS group, which was compared with the historical VPS controls in the SINPHONI study. Nonparametric statistical methods were used in all analyses. The Mann-Whitney U-test was used for comparisons between groups. Fisher's exact test was used to compare the 2 proportions. We compared the differences in the changes between the groups using analysis of covariance. The outcome variables at 6 months after randomization or the outcome variables at 12 months after surgery were entered in the model as a dependent variable. The baseline value of the outcome variable was included as a covariate, and the study group was included as a categorical variable. We calculated the $95 \%$ confidence intervals for proportions using the ClopperPearson binomial confidence method. The significance level was set at 2 -sided $p=0.05$. Statistical data were analyzed using SPSS version 22.

\section{Results \\ Trial Profile}

The first patient was recruited on March 1, 2010, and the follow-up for the last patient was completed on December 5, 2012. The flowchart for this study is shown in Fig. 1. Preregistration was completed for 102 patients. Of these patients, 49 were randomly assigned to the immediate group, and 44 to the postponed group. Three patients in the immediate group (1 diagnosed with pneumonia, 1 with burns, and 1 who withdrew consent) were excluded from analysis before LPS implantation, whereas 2 patients in the postponed group who withdrew consent were excluded before starting physical exercises. Thus, 88 patients were included in the FAS, of whom 46 were assigned to the immediate group and 42 to the postponed group. In the postponed group, 1 patient did not undergo shunt surgery because of an accumulation of ascites fluid of unknown etiology, whereas another patient was not evaluated after shunt surgery because of hospital transfer. Thus, the FAS of the postponed group after LPS implantation consisted of 40 patients. The central MRI review committee excluded 2 patients from the postponed group because they did not meet MRI criteria. Seven patients (5 in the immediate group and 2 in the postponed group) failed to undergo the 1-year final follow-up; of these patients, 6 patients remained in the PPS, with their scores inputted as LOCF. Thus, the PPS comprised 83 patients, of whom 45 belonged to the immediate group and 38 to the postponed group.

\section{Characteristics of Patients at Baseline}

The demographic characteristics of PPS patients are summarized in Table 1. Patients in SINPHONI-2 (the present study) were 2 years older than those in the SINPHONI study. There were no differences in functional status and symptoms between patients in the SINPHONI-2 and SINPHONI studies at baseline.

\section{Clinical Outcomes}

The mean overall initial valve pressure was $13.5 \pm 3.9$ $\mathrm{cm} \mathrm{H}_{2} \mathrm{O}$. This pressure was maintained throughout the follow-up period in 34 patients (40\%) and was readjusted in the other patients. Significant differences in $\mathrm{mRS}$ and iNPHGS gait scores were observed between the immediate and postponed groups during the 6-month period after randomization (Table 2). Although there was an improvement in mRS and iNPHGS gait scores over the 6-month period after randomization, scores were still higher in the postponed group than in the immediate group (Fig. 2). Furthermore, the proportion of patients with improvement in their mRS score was significantly higher in the immediate group $(65 \%, 95 \%$ CI $51 \%-79 \%)$ than in the postponed group $(29 \%, 95 \% \mathrm{CI} 14 \%-45 \% ; \mathrm{p}=0.027)$ at 6 months after randomization. Improvement in the postponed group was somewhat less than that in the immediate group at 6 months after randomization. 


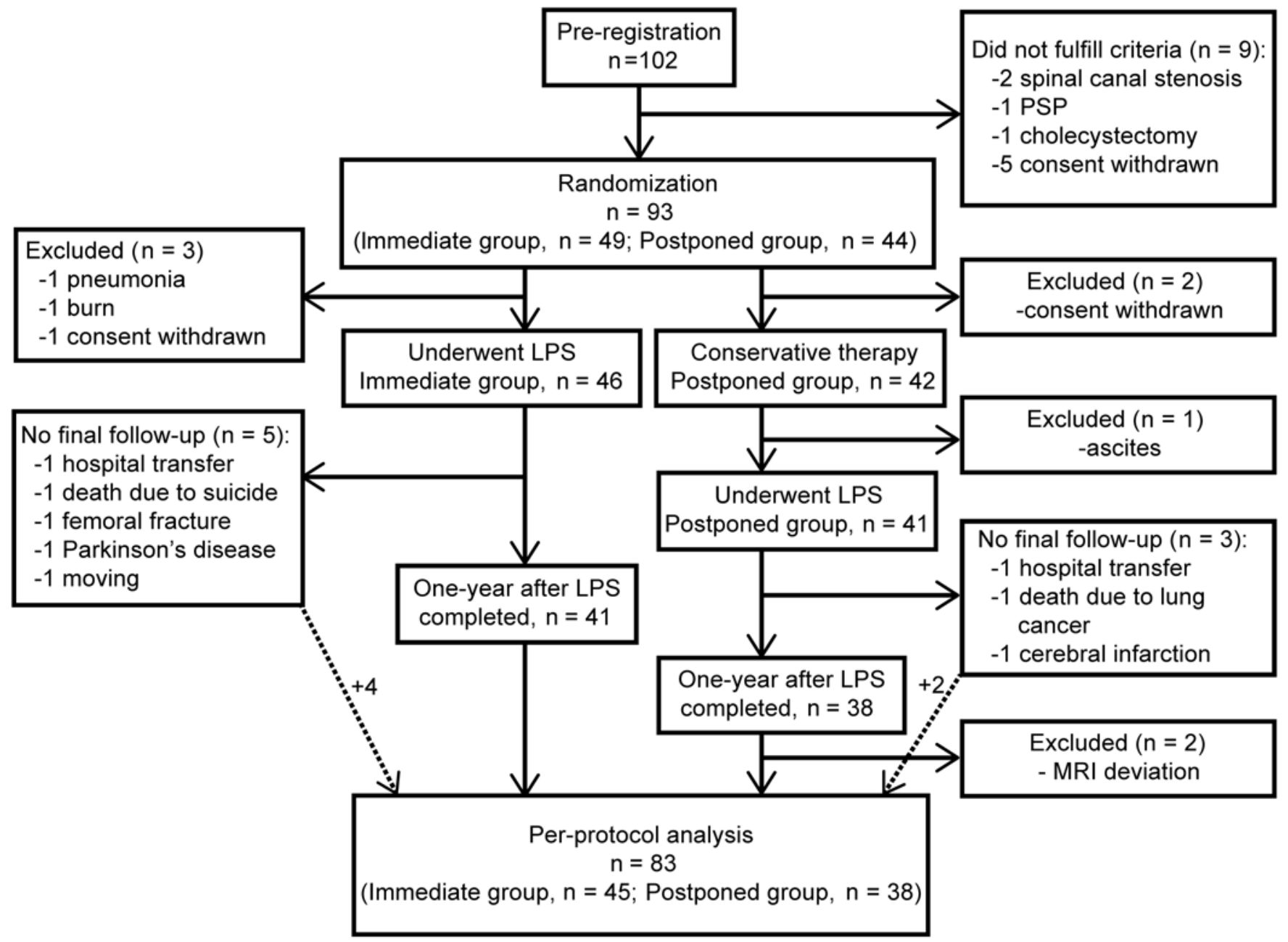

FIG. 1. Flowchart of the trial profile showing the number of patients from initial screening to final analysis. PSP = progressive supranuclear palsy.

There were no significant changes in mRS, iNPHGS, TUG, or MMSE scores between the immediate and postponed groups at 1 year after surgery (Table 3 ). A favorable outcome was achieved in $63 \%$ of patients (immediate group $67 \%$, postponed group 58\%; Table 4). The number of patients without functional impairment (mRS score < 1) increased from $1(1 \%)$ at preoperative assessment to $26(31 \%)$ by 1 year after surgery, whereas the number of independent patients ( $\mathrm{mRS}$ score $\leq 2)$ increased from 31 $(37 \%)$ at preoperative assessment to $53(63 \%)$ at 1 year after surgery (Fig. 3). When clinical improvement is defined as a decrease of 1 or more points in the total iNPHGS score, benefits occurred in $75 \%$ of patients (immediate group $80 \%$, postponed group $68 \%$ ) by 1 year after surgery. The proportion of shunt responders was $71 \%$ (immediate group $78 \%$, postponed group 63\%). Given that there was a significant difference in $\mathrm{mRS}$ scores between the 2 groups at 6 months after randomization, the recovery of the postponed group was delayed by the same interval of 6 months, although the scores in the postponed group almost caught up to those of the immediate group by 12 months after LPS implantation (Fig. 2).

\section{Adverse Events Associated With LPS Implantation}

The SAS included 87 patients from the SINPHONI-2 study. One patient in the postponed group was excluded from the analysis of adverse events at 1 year after LPS implantation because she did not receive an LPS. SAEs were observed in 19 patients, among whom death occurred in 2 patients (lung cancer and suicide). The outcome at 1 year after surgery was favorable in 9 of the patients and unfavorable in 10 patients (including the 2 deaths). Ten SAEs were directly related to surgery or the LPS: 3 subdural hematomas requiring surgery, 5 shunt tube migrations, 1 shunt tube rupture, and 1 case of meningitis. In addition, 29 nonserious, shunt-related adverse events were recorded in 24 patients: asymptomatic subdural fluid collection or hematoma was observed in brain imaging in 7 patients, and postural headache occurred in 21 patients; all of these adverse events were successfully controlled by the adjustment of valve pressure (Table 5).

\section{Comparison Between SINPHONI-2 and SINPHONI}

The proportion of favorable outcomes as measured 
TABLE 1. Patient characteristics at baseline: PPS analysis

\begin{tabular}{|c|c|c|c|c|c|}
\hline \multirow[b]{2}{*}{ Parameter } & \multicolumn{3}{|c|}{ SINPHONI-2 (LPS) } & \multirow[b]{2}{*}{ SINPHONI (VPS) } & \multirow[b]{2}{*}{$\mathrm{p}$ Value } \\
\hline & $\begin{array}{l}\text { Immediate } \\
\text { Surgery }\end{array}$ & $\begin{array}{l}\text { Postponed } \\
\text { Surgery }\end{array}$ & All & & \\
\hline No. of patients & 45 & 38 & 83 & 100 & \\
\hline \multicolumn{6}{|c|}{$\begin{array}{l}\text { Demographic details, no. (\%) unless } \\
\text { otherwise noted }\end{array}$} \\
\hline Mean age in yrs $\pm S D$ & $76.5 \pm 4.4$ & $76.3 \pm 5.0$ & $76.4 \pm 4.7$ & $74.5 \pm 5.1$ & 0.011 \\
\hline F/M sex & $27 / 18(60 / 40)$ & $11 / 27(29 / 71)$ & $38 / 45(46 / 54)$ & $42 / 58(42 / 58)$ & 0.655 \\
\hline History of hypertension & $27(60)$ & $20(53)$ & $47(57)$ & $60(60)$ & 0.655 \\
\hline History of diabetes & $10(22)$ & $10(26)$ & $20(24)$ & $20(20)$ & 0.591 \\
\hline History of lipid disorder & $15(33)$ & $13(34)$ & $28(34)$ & $31(31)$ & 0.752 \\
\hline \multicolumn{6}{|c|}{ Preop clinical findings, mean \pm SD } \\
\hline $\mathrm{mRS}$ & $2.8 \pm 0.8$ & $2.9 \pm 0.8$ & $2.9 \pm 0.8$ & $3.0 \pm 1.0$ & 0.218 \\
\hline Total iNPHGS & $6.5 \pm 2.0$ & $6.6 \pm 2.1$ & $6.6 \pm 2.4$ & $6.5 \pm 2.0$ & 0.941 \\
\hline Gait score & $2.3 \pm 0.7$ & $2.4 \pm 0.7$ & $2.4 \pm 0.7$ & $2.4 \pm 0.7$ & 0.878 \\
\hline Cognitive score & $2.3 \pm 0.9$ & $2.3 \pm 0.8$ & $2.3 \pm 0.9$ & $2.3 \pm 1.0$ & 0.939 \\
\hline Urinary score & $1.9 \pm 1.0$ & $1.9 \pm 1.1$ & $1.9 \pm 1.0$ & $1.9 \pm 1.3$ & 0.813 \\
\hline TUG & $34.8 \pm 39.8$ & $28.9 \pm 31.8$ & $32.1 \pm 36.5$ & $22.5 \pm 15.6$ & 0.534 \\
\hline MMSE & $20.3 \pm 6.1$ & $18.9 \pm 6.7$ & $19.6 \pm 6.4$ & $21.0 \pm 6.3$ & 0.093 \\
\hline \multicolumn{6}{|c|}{ Combinations of symptoms, no. (\%) } \\
\hline Triad & $25(56)$ & $20(53)$ & $45(54)$ & $51(51)$ & 0.990 \\
\hline Gait and cognitive & $8(18)$ & $9(24)$ & $17(20)$ & $23(23)$ & \\
\hline Gait and urinary & $2(4)$ & $1(3)$ & $3(4)$ & $5(5)$ & \\
\hline Cognitive and urinary & $2(4)$ & $1(3)$ & $3(4)$ & $3(3)$ & \\
\hline Gait only & $4(9)$ & $6(16)$ & $10(12)$ & $12(12)$ & \\
\hline Cognitive only & $3(7)$ & $1(3)$ & $4(5)$ & $3(3)$ & \\
\hline Urinary only & $0(0)$ & $1(3)$ & $1(1)$ & $1(1)$ & \\
\hline CSF pressure in $\mathrm{cm} \mathrm{H}_{2} \mathrm{O}^{*}$ & $12.5 \pm 2.9$ & $12.7 \pm 3.2$ & $12.6 \pm 3.0$ & $11.9 \pm 3.4$ & 0.303 \\
\hline
\end{tabular}

* The CSF pressure data correspond with opening pressure taken as the opening pressure at lumbar puncture.

by $\mathrm{mRS}$ and iNPHGS scores between the SINPHONI-2 and the SINPHONI study was comparable (Table 4). The changes in mRS scores at 12 months after surgery were not different from those in SINPHONI (Table 6). The proportion of patients with SAEs and non-SAEs did not differ significantly between SINPHONI-2 and SINPHONI at 1 year after surgery (SAEs: 19 [22\%] of 87 patients in the LPS group vs 15 [15\%] of 100 patients in the VPS group, $p=0.226$; non-SAEs: 24 [27.6\%] in the LPS group vs 20
[20\%] in the VPS group, $p=0.223$ ). Shunt failures requiring revision (6 [7\%] patients in the LPS group vs 1 [1\%] in the VPS group) and postural headache after surgery were more common with the use of LPS (Table 5). All postural headaches improved within 3 months of LPS surgery.

\section{Discussion}

The present study examined the usefulness of an MRI-

TABLE 2. Changes in clinical variables within the treatment groups at 6 months after randomization: PPS analysis

\begin{tabular}{|c|c|c|c|c|c|c|}
\hline \multirow[b]{2}{*}{ Variable } & \multicolumn{2}{|c|}{ Immediate Group } & \multicolumn{2}{|c|}{ Postponed Group } & \multirow[b]{2}{*}{ Difference $(95 \% \mathrm{Cl})$} & \multirow[b]{2}{*}{ p Value* } \\
\hline & No. & Mean (SD) & No. & Mean (SD) & & \\
\hline $\mathrm{mRS}$ & 45 & $-0.8(0.9)$ & 38 & $-0.3(0.5)$ & $0.5(0.2-0.8)$ & 0.005 \\
\hline Total iNPHGS & 45 & $-2.1(1.8)$ & 38 & $-1.4(1.9)$ & $0.8(0-1.5)$ & 0.056 \\
\hline Gait iNPHGS & 45 & $-0.8(0.8)$ & 38 & $-0.4(0.8)$ & $0.4(0-0.7)$ & 0.029 \\
\hline Cognitive iNPHGS & 45 & $-0.5(0.9)$ & 38 & $-0.3(0.6)$ & $0.2(-0.1$ to 0.6$)$ & 0.182 \\
\hline Urinary iNPHGS & 45 & $-0.7(1.1)$ & 38 & $-0.7(1.0)$ & $0.2(-0.2$ to 0.6$)$ & 0.400 \\
\hline TUG in sec & 45 & $-13.8(35.4)$ & 37 & $-7.8(16.4)$ & $8.0(-8.2$ to 9.7$)$ & 0.868 \\
\hline MMSE & 45 & $1.4(3.7)$ & 38 & $1.6(3.6)$ & $-0.1(-1.7$ to 1.5$)$ & 0.932 \\
\hline
\end{tabular}

* Analysis of covariance: comparison between immediate and postponed groups, adjusted by baseline value. 


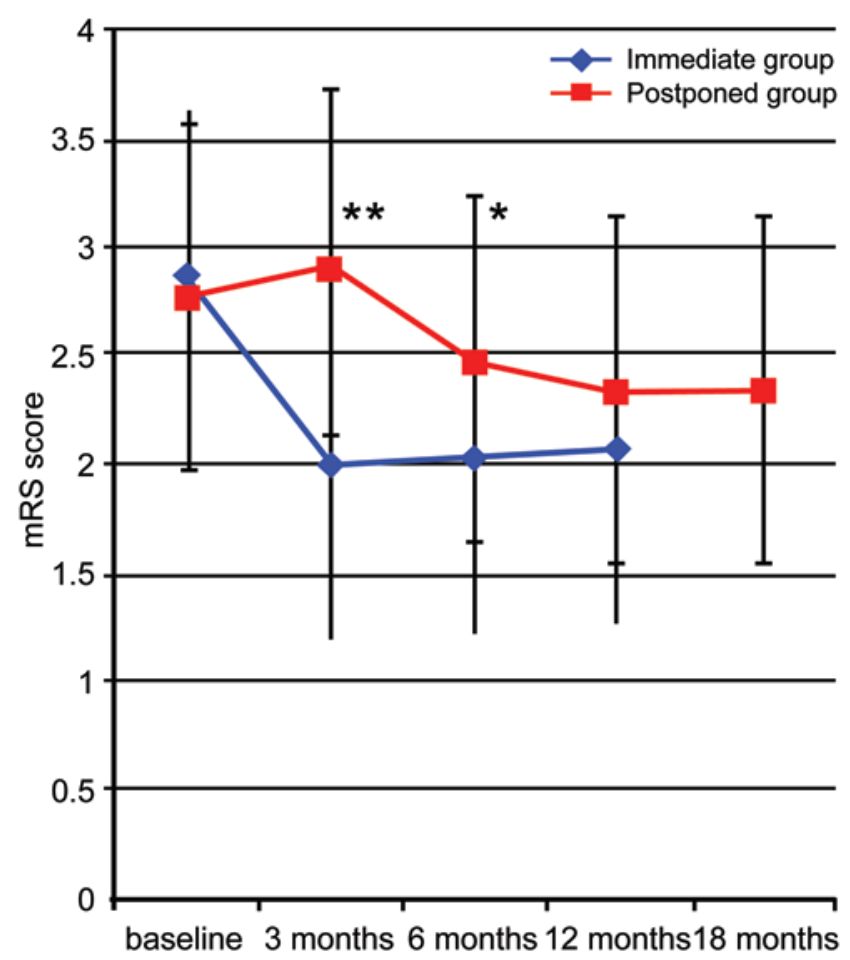

FIG. 2. Change in the mRS score between randomization and 1 year after LPS implantation. Error bars indicate the standard deviation. ${ }^{*} \mathrm{p}<$ $0.05,{ }^{* *} p<0.0001$. Figure is available in color online only.

based diagnostic scheme and the 1-year beneficial effect of LPS implantation in patients with iNPH. The most relevant finding of the present study was that $63 \%$ of patients with iNPH, who were diagnosed and referred for shunts based on clinical and MRI findings, experienced improved outcomes at 12 months after LPS implantation, as measured by $\mathrm{mRS}$ scores. The corresponding improvement rate was $75 \%$ when measured using iNPHGS scores. After VPS surgery, $69 \%$ of patients showed improvement at 1 year as measured by mRS scores, a proportion identical to the results from 2 previously reported European and Japanese (SINPHONI) multicenter studies. ${ }^{8,20}$ Compared with these 2 previous studies, there was no significant difference in ADL improvement between VPS and LPS. In addition, the SINPHONI trial reported an improvement of
$77 \%$ at 1 year after VPS implantation, as measured by the same iNPHGS used here. The present study showed that by 1 year after LPS implantation, clinical improvement of iNPH was nearly on par with that achieved using a VPS (Table 4). Actual changes in the mRS and iNPHGS scores in the SINPHONI-2 trial were comparable to those in the SINPHONI trial (Table 6).

The initial progression of iNPH symptoms is extremely gradual; therefore, the present study was planned on the assumption that a 3-month change in the procedure would have no impact on the efficacy of the surgery. However, contrary to what was expected and although clinical symptoms did not worsen in the 3-month period following diagnosis, by 1 year after surgery the postponed group showed improvement in ADLs and in symptoms that, while not significantly different from those in the immediate group, had decreased. It thus became clear that discovery of iNPH is difficult because of the extremely gradual progression of symptoms and because therapeutic efficacy decreases with delays in treatment. Recently, Andrén and colleagues reported that the natural course of $\mathrm{iNPH}$ is a progression of symptoms over time, with worsening gait, balance, and cognitive symptoms. ${ }^{4}$ Moreover, this deterioration is only partially reversible. Therefore, the authors recommended performing surgery soon after diagnosis to maximize the benefits of shunt treatment.

We observed SAEs in 19 patients and shunt failures requiring revision in 6 patients. Although the SINPHONI study reported SAEs in 15 patients, only 1 patient required revision because of ventricular shunt tube obstruction. Shunt failures directly related to LPS included 4 patients with a prolapse of the peritoneal catheter from the abdominal cavity and 1 patient with a lumbar catheter migration from the spinal canal. Conversely, the SINPHONI study using VPS found no cases of prolapse of the peritoneal catheter from the abdominal cavity ${ }^{8}$ (Table 5). In a study in which Bloch and McDermott used LPSs for the treatment of iNPH, there was 1 reported case of peritoneal catheter migration out of 33 patients, which indicates that the peritoneal catheter is more prone to prolapse after LPS implantation than after VPS insertion. ${ }^{6}$ This complication must be taken into consideration and is thought to be related to the lateral position of the patient during the operation as well as the abdominal subcutaneous adipose tissue content of the patient. Such shunt catheter migration can be avoided by using a recently developed surgical

TABLE 3. Changes in clinical variables within the treatment groups 1 year after surgery: PPS analysis

\begin{tabular}{|c|c|c|c|c|c|c|}
\hline \multirow[b]{2}{*}{ Variable } & \multicolumn{2}{|c|}{ Immediate Group } & \multicolumn{2}{|c|}{ Postponed Group } & \multirow[b]{2}{*}{ Difference $(95 \% \mathrm{Cl})$} & \multirow[b]{2}{*}{ p Value* } \\
\hline & No. & Mean (SD) & No. & Mean (SD) & & \\
\hline $\mathrm{mRS}$ & 45 & $-0.8(1.1)$ & 38 & $-0.6(0.7)$ & $0.2(-0.2$ to 0.6$)$ & 0.337 \\
\hline Total iNPHGS & 45 & $-1.9(2.6)$ & 38 & $-1.6(1.7)$ & $0.3(-0.6$ to 1.3$)$ & 0.483 \\
\hline Gait iNPHGS & 45 & $-0.8(1.0)$ & 38 & $-0.6(0.9)$ & $0.2(-0.2$ to 0.6$)$ & 0.348 \\
\hline Cognitive iNPHGS & 45 & $-0.6(1.0)$ & 38 & $-0.4(0.7)$ & $0.2(-0.2$ to 0.6$)$ & 0.340 \\
\hline Urinary iNPHGS & 45 & $-0.7(1.1)$ & 38 & $-0.6(0.9)$ & $0.1(-0.3$ to 0.5$)$ & 0.716 \\
\hline TUG in sec & 37 & $-13.5(41.3)$ & 35 & $-9.0(20.0)$ & $-3.1(-10.8$ to 4.5$)$ & 0.416 \\
\hline MMSE & 40 & $2.6(5.8)$ & 36 & $1.2(2.8)$ & $-1.8(-3.8$ to 0.2$)$ & 0.071 \\
\hline
\end{tabular}

* Analysis of covariance: comparison between immediate and postponed groups, adjusted by baseline value. 
TABLE 4. Patient improvement of 1 point or more on $m R S$ and iNPHGS 1 year after surgery: PPS analysis

\begin{tabular}{|c|c|c|c|c|c|c|c|c|c|}
\hline \multirow[b]{3}{*}{ Parameter } & \multicolumn{6}{|c|}{ SINPHONI-2 } & & & \multirow[b]{3}{*}{$\mathrm{p}$ Value } \\
\hline & \multicolumn{2}{|c|}{ Immediate Group } & \multicolumn{2}{|c|}{ Postponed Group } & \multicolumn{2}{|c|}{ All } & \multicolumn{2}{|c|}{ SINPHONI* } & \\
\hline & No. $(\%)$ & $95 \% \mathrm{Cl}$ & No. $(\%)$ & $95 \% \mathrm{Cl}$ & No. $(\%)$ & $95 \% \mathrm{Cl}$ & No. $(\%)$ & $95 \% \mathrm{Cl}$ & \\
\hline No. of patients & 45 & & 38 & & 83 & & 100 & & \\
\hline mRS (favorable outcomef) & $30(67)$ & $51-80$ & $22(58)$ & $41-74$ & $52(63)$ & $51-73$ & $69(69)$ & $59-78$ & 0.496 \\
\hline mRS (shunt responder§) & $35(78)$ & $63-89$ & $24(63)$ & $46-78$ & $59(71)$ & $60-81$ & $80(80)$ & $71-87$ & 0.155 \\
\hline iNPHGS (favorable outcome) & $36(80)$ & $65-90$ & $26(68)$ & $51-83$ & $62(75)$ & $64-84$ & $77(77)$ & $68-84$ & 0.312 \\
\hline iNPHGS (shunt responder) & $40(89)$ & $76-96$ & $31(82)$ & $66-92$ & $71(86)$ & $76-92$ & $89(89)$ & $81-94$ & 0.368 \\
\hline
\end{tabular}

technique, ${ }^{17}$ but further efforts to prevent shunt failure are required. Orthostatic headaches (a complication of excessive shunting of spinal fluid) were observed during the perioperative period in 21 patients, and subdural effusion or hematomas were observed in 10 patients. Of these 10 patients, remedial bur hole surgery was performed in 3. In the early postoperative period, reported problems unique to LPSs included spinal fluid leakage from the location of the access site into the epidural space. When postoperative orthostatic headaches are observed, the shunt pressure setting must be set to high pressure, and the patient must rest in a recumbent position until the headache subsides. ${ }^{14,23}$

In a systematic review by Hebb and Cusimano, the overall complication rate was $38 \%$, with $22 \%$ of patients requiring additional surgery. ${ }^{9}$ Even with the use of programmable valves, modern iNPH series have reported nontraumatic subdural hematoma rates as high as $10 \%$, with up to $7 \%$ requiring surgical evacuation. ${ }^{20,24,34}$ We used programmable valves with an antisiphon device in the present study,

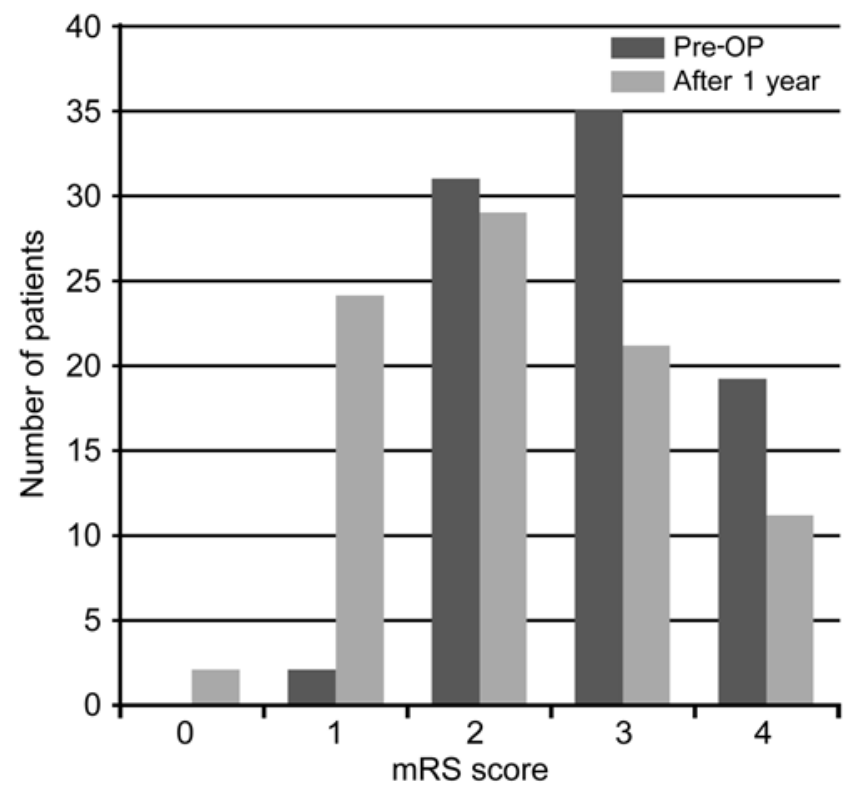

FIG. 3. Bar graph depicting the functional status of patients based on mRS scores prior to shunt implantation (Pre-OP) and at 1 year after LPS placement. which may have resulted in 3 cases $(3 \%)$ of subdural hematoma that required surgery, resulting in lower revision rates. Compared with rates in previous studies, the complication rate in the present study is acceptable.

We are aware of the criticism of LPSs in European and North American countries with regard to the higher revision rate than that with VPSs. Given this criticism, an

TABLE 5. Adverse events

\begin{tabular}{|c|c|c|}
\hline Parameter & SINPHONI-2 & SINPHONI* \\
\hline Total no. of patients & 87 & 100 \\
\hline \multicolumn{3}{|l|}{ SAEs } \\
\hline No. of patients & $19(22 \%)$ & $15(15)$ \\
\hline Total no. of events & 21 & 17 \\
\hline $\begin{array}{l}\text { Subdural hematoma requiring sur- } \\
\text { gery }\end{array}$ & $3(3.4 \%)$ & $1(1 \%)$ \\
\hline $\begin{array}{l}\text { Shunt tube migration requiring re- } \\
\text { vision }\end{array}$ & $5(5.7 \%)$ & \\
\hline Shunt tube rupture requiring revision & $1(1.1 \%)$ & \\
\hline Shunt tube obstruction & & $1(1 \%)$ \\
\hline Bowel perforation & & $1(1 \%)$ \\
\hline Meningitis & $1(1.1 \%)$ & \\
\hline Pneumonia & & $3(3 \%)$ \\
\hline Malignancy & & $2(2 \%)$ \\
\hline Head injury & & $1(1 \%)$ \\
\hline Cerebral infarction & $6(6.9 \%)$ & $3(3 \%)$ \\
\hline Myocardial infarction & & $1(1 \%)$ \\
\hline Femoral fracture & $2(2.3 \%)$ & $2(2 \%)$ \\
\hline $\begin{array}{l}\text { Lumbar vertebral compression frac- } \\
\text { ture }\end{array}$ & $1(1.1 \%)$ & \\
\hline Death & $2(2.3 \%)$ & $2(2 \%)$ \\
\hline \multicolumn{3}{|l|}{ Non-SAE } \\
\hline No. of patients & $24(27.6 \%)$ & $20(20 \%)$ \\
\hline Total no. of events & 29 & $21(21 \%)$ \\
\hline Postural headache & $21(24.1 \%)$ & $8(8 \%)$ \\
\hline Asymptomatic subdural effusion & $5(5.7 \%)$ & $8(8 \%)$ \\
\hline Subdural hematoma conservative & $2(2.3 \%)$ & $5(5 \%)$ \\
\hline Bronchial asthma & $1(1.1 \%)$ & \\
\hline
\end{tabular}

* SINPHONI data (i.e., historical control) extracted from Hashimoto et al. ${ }^{8}$ 
TABLE 6. Changes in clinical variables within the 2 studies 1 year after surgery (PPS analysis)

\begin{tabular}{|c|c|c|c|c|}
\hline \multirow[b]{2}{*}{ Variable } & \multicolumn{2}{|c|}{ SINPHONI-2 } & \multicolumn{2}{|c|}{ SINPHONI* $^{*}$} \\
\hline & No. & Difference $(95 \% \mathrm{Cl})$ & No. & Difference $(95 \% \mathrm{Cl})$ \\
\hline \multicolumn{5}{|l|}{$\mathrm{mRS}$} \\
\hline All & 83 & $-0.7(-0.9$ to -0.5$)$ & & \\
\hline Immediate & 45 & $-0.8(-1.1$ to -0.5$)$ & 100 & $-1.0(-1.2$ to -0.8$)$ \\
\hline Postponed & 38 & $-0.6(-0.8$ to -0.3$)$ & & \\
\hline \multicolumn{5}{|l|}{ iNPHGS } \\
\hline Total score & 83 & $-1.7(-2.2$ to -1.2$)$ & 100 & $-2.6(-3.1$ to -2.1$)$ \\
\hline Gait score & 83 & $-0.7(-0.9$ to -0.5$)$ & 100 & $-1.0(-1.2$ to -0.8$)$ \\
\hline Cognitive score & 83 & $-0.4(-0.7$ to -0.3$)$ & 100 & $-0.8(-1.2$ to -0.8$)$ \\
\hline Urinary score & 83 & $-0.7(-0.9$ to -0.4$)$ & 100 & $-0.9(-1.0$ to -0.7$)$ \\
\hline TUG in sec & 72 & $-11.3(-18.8$ to -3.8$)$ & 80 & $-7.7(-11.0$ to -4.3$)$ \\
\hline MMSE & 76 & $1.9(0.9$ to 3.0$)$ & 98 & 2.2 (1.2 to 3.2 ) \\
\hline
\end{tabular}

* SINPHONI data (i.e., historical control) extracted from Hashimoto et al. ${ }^{8}$

LPS is not commonly used for the treatment of iNPH, but rather has been primarily adopted for treating idiopathic intracranial hypertension, a disease that tends to occur in younger patients. ${ }^{1,31,33}$ Despite the unfavorable view of LPSs in European and North American countries, we adopted the use of LPSs in the present study. The following reasons provide the rationale behind this decision. First, one of the advantages of an LPS is that surgical procedures can be performed exclusively extracranially, minimizing intracranial complications. Second, the prevalence of obesity in the Japanese population (body mass index [BMI] in this study $23.8 \pm 3$ ) is dramatically lower than that in European and North American studies (BMI 2628). ${ }^{13,22,28}$ This may have played an important role in preventing malfunction of the LPS by keeping the abdominal catheter in place.

The present study has several notable limitations that require discussion. The most critical issue is that the study lacked a direct comparison with VPSs. Therefore, data from the SINPHONI study were used as a historical control. A second crucial issue is the nonblinded design of the study: neurosurgeons and neurologists in charge of patient care and evaluation were aware of the treatment, which represents a possible source of performance and detection bias. Third, the inclusion criteria were based on the diagnostic criteria of the Japanese iNPH guidelines, which are somewhat different from the criteria of the international iNPH guidelines. These issues should be taken into consideration before the findings are generalized. Moreover, our follow-up period was limited to 1 year. To clearly define the benefit of LPSs, this period should be extended in future trials. In addition, we did not evaluate the effect of LPSs on the health-related quality of life of patients, which needs to be addressed in further studies.

\section{Conclusions}

The efficacy and safety of LPSs with programmable valves are comparable to those of VPSs for the treatment of patients with iNPH. Because LPSs are still not widely used for the treatment of iNPH, LPSs must be technically improved to prevent shunt failure. Despite its relatively high shunt failure rate, an LPS is the treatment of choice because of its minimal invasiveness and avoidance of brain injury in elderly patients.

\section{Appendix \\ Location of Work}

SINPHONI-2 was a project of the Japanese Society of NormalPressure Hydrocephalus and involved 20 centers in Japan.

\section{SINPHONI-2 Investigators}

Principal Investigators

Masatsune Ishikawa and Etsuro Mori.

\section{Steering Committee}

M. Ishikawa, E. Mori, Masakazu Miyajima, and Hiroaki Kazui.

\section{Writing Committee}

Hiroaki Kazui, Etsuro Mori, Masatsune Ishikawa, and Masakazu Miyajima.

\section{Study Advisory Board}

Osamu Hirai (Shinko Hospital), Nobumasa Kuwana (Tokyo Kyosai Hospital), Masaaki Hashimoto (Noto General Hospital), Kazunari Ishii (Kinki University School of Medicine), Yoshihumi Hirata (Kumamoto Takumadai Hospital), Hiroji Miyake (Nishinomiya Kyoritsu Neurosurgical Hospital), Satoru Mori (University of Shiga Prefecture), and Yoshinaga Kajimoto (Osaka Medical College).

\section{Study Statistician}

Hideki Oligasa (Professor, Department of Biostatistics and Clinical Epidemiology, Graduate School of Medicine and Pharmaceutical Sciences, University of Toyama).

\section{Independent Data and Safety Monitoring Committee}

Haruko Yamamoto (Chairperson, Director, Department of Clinical Research, National Cerebral and Cardiovascular Center), Koreaki Mori (Professor Emeritus, Kochi Medical School), Shigenobu Nakamura (Director, Rakuwakai Kyoto Clinical Research Center), Tamotsu Miki (Professor, Department of Neurosurgery, Tokyo Medical University). 


\section{Investigators and Centers}

Masatsune Ishikawa (Rakuwakai Otowa Hospital, Kyoto); Etsuro Mori (Tohoku University Graduate School of Medicine, Sendai); Hajime Arai and Masakazu Miyajima (Juntendo University Graduate School of Medicine, Tokyo); Masaaki Hashimoto (Noto General Hospital, Nanao); Yoshinaga Kajimoto (Osaka Medical Collage, Takatsuki); Hiroji Miyake (Nishinomiya Kyoritsu Neurosurgical Hospital, Nishinomiya); Teiji Nakayama (Hamamatsu Medical Center, Hamamatsu); Osamu Hirai (Shinko Hospital, Kobe); Hiroaki Kazui and Masatoshi Takeda (Osaka University Graduate School of Medicine, Suita); Nobumasa Kuwana (Tokyo Kyosai Hospital, Tokyo); Chia-Cheng Chang (Yokohama Minami Kyosai Hospital, Yokohama); Isao Date (Okayama University Graduate School of Medicine, Okayama); Yoshihumi Hirata (Kumamoto Takumadai Hospital, Kumamoto); Masamichi Atsuchi (Atsuchi Neurosurgical Hospital, Kagoshima); Takaharu Okada (Tama-Hokubu Medical Center, Higashimurayama); Junichiro Hamada (Kanazawa University Graduate School of Medicine, Kanazawa); Mitsuya Watanabe (Tama-Nanbu Regional Hospital, Tama); Teruo Kimura (Dohtoh Neurosurgical Hospital, Kitami); Mitsunobu Kaijima (Megumino Hospital, Eniwa); Souichi Sunada (Tsudanuma Central General Hospital, Narashino).

\section{Acknowledgments}

This study was a project of the Japanese Society of NormalPressure Hydrocephalus. We thank the patients for their participation. We also thank the people who agreed to participate in this trial and the study contributors.

\section{References}

1. Abubaker K, Ali Z, Raza K, Bolger C, Rawluk D, O'Brien D: Idiopathic intracranial hypertension: lumboperitoneal shunts versus ventriculoperitoneal shunts-case series and literature review. Br J Neurosurg 25:94-99, 2011

2. Adams RD, Fisher CM, Hakim S, Ojemann RG, Sweet WH: Symptomatic occult hydrocephalus with normal cerebrospinal-fluid pressure: a treatable syndrome. N Engl J Med 273:117-126, 1965

3. Akiguchi I, Shirakashi Y, Budka H, Watanabe Y, Watanabe T, Shiino A, et al: Disproportionate subarachnoid space hydrocephalus-outcome and perivascular space. Ann Clin Transl Neurol 1:562-569, 2014

4. Andrén K, Wikkelsø C, Tisell M, Hellström P: Natural course of idiopathic normal pressure hydrocephalus. J Neurol Neurosurg Psychiatry 85:806-810, 2014

5. Bergsneider M, Black PM, Klinge P, Marmarou A, Relkin N: Surgical management of idiopathic normal-pressure hydrocephalus. Neurosurgery 57 (3 Suppl):S29-S39, ii-v, 2005

6. Bloch O, McDermott MW: Lumboperitoneal shunts for the treatment of normal pressure hydrocephalus. J Clin Neurosci 19:1107-1111, 2012

7. Eide PK, Sorteberg W: Diagnostic intracranial pressure monitoring and surgical management in idiopathic normal pressure hydrocephalus: a 6 -year review of 214 patients. Neurosurgery 66:80-91, 2010

8. Hashimoto M, Ishikawa M, Mori E, Kuwana N: Diagnosis of idiopathic normal pressure hydrocephalus is supported by MRI-based scheme: a prospective cohort study. Cerebrospinal Fluid Res 7:18, 2010

9. Hebb AO, Cusimano MD: Idiopathic normal pressure hydrocephalus: a systematic review of diagnosis and outcome. Neurosurgery 49:1166-1186, 2001

10. Hiraoka K, Meguro K, Mori E: Prevalence of idiopathic normal-pressure hydrocephalus in the elderly population of a Japanese rural community. Neurol Med Chir (Tokyo) 48:197-200, 2008
11. Iseki C, Kawanami T, Nagasawa H, Wada M, Koyama S, Kikuchi K, et al: Asymptomatic ventriculomegaly with features of idiopathic normal pressure hydrocephalus on MRI (AVIM) in the elderly: a prospective study in a Japanese population. J Neurol Sci 277:54-57, 2009

12. Jaraj D, Rabiei K, Marlow T, Jensen C, Skoog I, Wikkelsø C: Prevalence of idiopathic normal-pressure hydrocephalus. Neurology 82:1449-1454, 2014

13. Jusué-Torres I, Hoffberger JB, Rigamonti D: Complications of lumboperitoneal shunts for normal pressure hydrocephalus. Cureus 6:e171, 2014

14. Kaijima M, Fukuda H, Yamamoto K. [Post-operative complications peculiar to lumboperitoneal shunt: possible consequences due to side leakage of CSF from around the inserted spinal tube into the lumbar epidural space.] No Shinkei Geka 39:497-504, 2011 (Jpn)

15. Kanazawa R, Ishihara S, Sato S, Teramoto A, Kuniyoshi N: Familiarization with lumboperitoneal shunt using some technical resources. World Neurosurg 76:347-351, 2011

16. Karabatsou K, Quigley G, Buxton N, Foy P, Mallucci C: Lumboperitoneal shunts: are the complications acceptable? Acta Neurochir (Wien) 146:1193-1197, 2004

17. Kawahara T, Tokimura H, Higa N, Hirano H, Bohara M, Hanaya R, et al: Surgical technique for preventing subcutaneous migration of distal lumboperitoneal shunt catheters. Innovative Neurosurgery 1:169-172, 2013

18. Kazui H, Miyajima M, Mori E, Ishikawa M: Lumboperitoneal shunt surgery for idiopathic normal pressure hydrocephalus (SINPHONI-2): an open-label randomised controlled trial. Lancet Neurol 14:585-594, 2015

19. Kitagaki H, Mori E, Ishii K, Yamaji S, Hirono N, Imamura T: CSF spaces in idiopathic normal pressure hydrocephalus: morphology and volumetry. AJNR Am J Neuroradiol 19:1277-1284, 1998

20. Klinge P, Hellström P, Tans J, Wikkels $\varnothing$ C: One-year outcome in the European multicentre study on iNPH. Acta Neurol Scand 126:145-153, 2012

21. Kubo Y, Kazui H, Yoshida T, Kito Y, Kimura N, Tokunaga $\mathrm{H}$, et al: Validation of grading scale for evaluating symptoms of idiopathic normal-pressure hydrocephalus. Dement Geriatr Cogn Disord 25:37-45, 2008

22. Lemcke J, Meier U, Müller C, Fritsch MJ, Kehler U, Langer N, et al: Safety and efficacy of gravitational shunt valves in patients with idiopathic normal pressure hydrocephalus: a pragmatic, randomised, open label, multicentre trial (SVASONA). J Neurol Neurosurg Psychiatry 84:850-857, 2013

23. Matsubara T, Ishikawa E, Hirata K, Matsuda M, Akutsu H, Masumoto T, et al: A new mechanism of cerebrospinal fluid leakage after lumboperitoneal shunt: a theory of shunt side hole-case report. Neurol Med Chir (Tokyo) 54:572-577, 2014

24. McGirt MJ, Woodworth G, Coon AL, Thomas G, Williams MA, Rigamonti D: Diagnosis, treatment, and analysis of long-term outcomes in idiopathic normal-pressure hydrocephalus. Neurosurgery 57:699-705, 2005

25. Miyake H, Kajimoto Y, Murai H, Nomura S, Ono S, Okamoto Y, et al: Assessment of a quick reference table algorithm for determining initial postoperative pressure settings of programmable pressure valves in patients with idiopathic normal pressure hydrocephalus: SINPHONI subanalysis. Neurosurgery 71:722-728, 2012

26. Nakajima M, Miyajima M, Ogino I, Sugano H, Akiba C, Domon N, et al: Use of external lumbar cerebrospinal fluid drainage and lumboperitoneal shunts with Strata NSC valves in idiopathic normal pressure hydrocephalus: a single-center experience. World Neurosurg 83:387-393, 2015

27. Podsiadlo D, Richardson S: The timed "Up \& Go": a test of basic functional mobility for frail elderly persons. J Am Geriatr Soc 39:142-148, 1991 
28. Sæhle T, Farahmand D, Eide PK, Tisell M, Wikkelsö C: A randomized controlled dual-center trial on shunt complications in idiopathic normal-pressure hydrocephalus treated with gradually reduced or "fixed" pressure valve settings. J Neurosurg 121:1257-1263, 2014

29. Tanaka N, Yamaguchi S, Ishikawa H, Ishii H, Meguro K: Prevalence of possible idiopathic normal-pressure hydrocephalus in Japan: the Osaki-Tajiri project. Neuroepidemiology 32:171-175, 2009

30. Taylor AR: Fallacies in interpretation of Queckenstedt's test Lancet 2:1001-1004, 1960

31. Toma AK, Dherijha M, Kitchen ND, Watkins LD: Use of lumboperitoneal shunts with the Strata NSC valve: a singlecenter experience. J Neurosurg 113:1304-1308, 2010

32. van Swieten JC, Koudstaal PJ, Visser MC, Schouten HJ, van Gijn J: Interobserver agreement for the assessment of handicap in stroke patients. Stroke 19:604-607, 1988

33. Wang VY, Barbaro NM, Lawton MT, Pitts L, Kunwar S, Parsa AT, et al: Complications of lumboperitoneal shunts. Neurosurgery 60:1045-1049, 2007

34. Zemack G, Romner B: Adjustable valves in normal-pressure hydrocephalus: a retrospective study of 218 patients. Neurosurgery 51:1392-1402, 2002

\section{Disclosures}

Dr. Miyajima receives clinical and research support from
Johnson and Johnson K.K., Inc. (Japan) and Nihon Medi-Physics Company, Ltd. (Japan). Dr. Ishikawa receives lecture fees from Codman and Shurtleff (Japan) and Medtronic Corp. (Japan). Dr. Mori receives honoraria from Johnson and Johnson K.K., Inc. Dr. Kazui receives honoraria and accepted donations for the 15 th Japan Congress of Normal Pressure Hydrocephalus from Johnson and Johnson K.K., Inc.

\section{Author Contributions}

Conception and design: all authors. Acquisition of data: Miyajima, Kazui, Ishikawa. Analysis and interpretation of data: all authors. Drafting the article: Miyajima. Critically revising the article: all authors. Reviewed submitted version of manuscript: all authors. Approved the final version of the manuscript on behalf of all authors: Miyajima. Statistical analysis: Miyajima, Mori. Administrative/technical/material support: Kazui. Study supervision: Mori, Ishikawa.

\section{Correspondence}

Masakazu Miyajima, Department of Neurosurgery, Juntendo University Graduate School of Medicine, 2-1-1, Hongo, Bunkyou-ku, Tokyo 113-8421, Japan.email: mmasaka@ juntendo.ac.jp. 